\title{
Frozen-to-jamming-to-fluid Transition of Weakly Sheared Granular Systems by Low- frequency Mechanical Spectroscopy
}

\author{
Hanyu Tang ${ }^{a}$, Na Wang ${ }^{a}$, Changsong Liu ${ }^{a *}$, Xuebang $\mathrm{Wu}^{a}$ (®) \\ ${ }^{a}$ Key Laboratory of Materials Physics, Institute of Solid State Physics, Chinese Academy of Sciences, \\ P.O. Box 1129, Hefei 230031, P.R. China
}

Received: September 29, 2017; Revised: May 03, 2018; Accepted: May 24, 2018

\begin{abstract}
Granular matter usually displays frozen, jamming and fluidized states when submitted to an external vibration with increasing intensity. The dissipation properties of granular systems with three different millimeter-size glass grains $(0.1,0.5$ and $1.9 \mathrm{~mm})$ have been investigated by a modified lowfrequency inverted torsion pendulum under a shear strain and an external pressure. With increasing the immersed depth of the oscillating probe, all the systems show the frozen, jamming and fluidized behaviors. Furthermore, the critical depth at which the transition occurs increases with increasing grain size, but decreases with the application of pressure. A qualitative explanation is tentatively proposed to understand the underlying mechanism of complex viscoelastic properties of the glass particle systems.
\end{abstract}

Keywords: granular matter, jamming, mechanical spectroscopy.

\section{Introduction}

Granular materials consist of a collection of macroscopic discrete particles interacting solely via contact forces ${ }^{1-3}$. The mechanical response of granular packings to external perturbations plays an important role in numerous engineering applications such as soil mechanics and geophysics as well as in industry ${ }^{4}$. Due to its rich phenomenology and important applications, the granular matter physics attracts a growing interest in past decades for addressing open problems of non-equilibrium mechanics such as the nature of slow glassy dynamics and jamming ${ }^{5}$. Perhaps the simplest example of such a system is a dense packing of spherical glass beads under pressure, where the pressure at first monotonically increases with the depth and then saturates after a few layers by the Janssen model ${ }^{6}$. Their physical behaviors involve complex nonlinear phenomena, such as energy dissipation and nonlinear elastic response, and have received much attention ${ }^{4}$.

By changing the external conditions, the frictional granular packing exhibits a complex jamming phase diagram and it shows several rheological regions, ranging from fluid-like (if strongly vibrated), to jamming (if weakly vibrated), to aging when they become trapped in frozen states ${ }^{7-16}$. At higher vibration intensities, the granular medium is fluidized. As the intensity is lowered below the fluidization limit, the jamming region is reached, where the network of force chains becomes more complex and the dynamics is controlled by rarer and rarer events ${ }^{13}$. In this situation, a behavior similar to a constrained random walk is obtained 14,15 . By investigating the mechanical properties of vibrated millimeter sized glass beads with an immersed torsion oscillator, A relaxation peak was observed and understood in terms of a thermal-like activated phenomenon indicative of glass transition arising from jamming ${ }^{16}$, as seen in usual glass-forming liquids such as polymers and colloids ${ }^{17-19}$. As the vibration intensity further decreases, the system evolves into a completely frozen state, where the system is too jammed to make the rearrangement of force chains and reached a spatially disordered static configuration with all the particles at rest ${ }^{14,15}$. Xiong et al. studied the elasticity of glassy beads under shear oscillation strain by examining the change of system resonant frequency, and deduced that the dynamics of system displays four different states: elastic solid, plastic solid, viscoelastic solid and viscous fluid state respectively ${ }^{20}$. By using a low-frequency inverted torsion pendulum immersed into granular materials, Wang et al. investigated the friction and relative energy dissipation in weakly sheared granular materials ${ }^{21}$. The dissipation mechanism was uncovered by measurements of the relative energy dissipation versus the amplitude and the immersed depth, and explained by a rheological model ${ }^{21}$. Recently, Sellerio et al. studied the dynamics of vibrated granular media with increasing agitation intensity and proposed a possible phase diagram, which shows the four dynamic regimes, that is frozen, jamming, fluidization and fluctuation dissipative regions ${ }^{15}$. Chai et al. ${ }^{22}$ studied the elastic properties of grain chains by measuring the resonance dissipation peaks in different grain systems under weak vibrations and low pressures. A universal quantitative relationship between the resonant frequency of grain chains and the deformation is shown for different granular materials under pressures ${ }^{22}$.

These studies have certainly advanced our understanding of the mechanical properties and dynamics of granular materials, however, the underlying mechanism of the complex dynamics of granular assemblies are still not completely understood, especially on the knowledge of the effects of 
granular size and the external pressure on the dynamics. In this work, we investigated the frozen, jamming and fluidized transitions of glass bead packings with three different millimeter size grains $(0.1,0.5$ and $1.9 \mathrm{~mm})$ by monitoring viscoelastic behaviors with a low-frequency mechanical spectroscopy under a shear strain and an external pressure. The systems exhibit a frozen-to-jamming-to-fluid transition with increasing the immersed depth of the oscillating probe. It is found that the jamming transition of the system are very strongly dependent on the grain size and the external pressure. A qualitative explanation is proposed to interpret the experimental findings of the granular systems.

\section{Experiment}

The inverted torsion pendulum (see Fig. 1) consists of a cylindrical probe that is able to rotate around its axis, but (to be) prevented from moving sideways by a steel wire fixed to the two ends of the probe. The rotating probe of a torsion oscillator is immersed into the granular material. The probe is forced into torsional vibration by a time-dependent force $F(t)=F \sin (\omega t)$, exerted by applying a pair of permanent magnets fixed to the pendulum and external coils. The angular displacement function of the probe, $A(t)$, is measured optically. In the case here, the response of the argument is

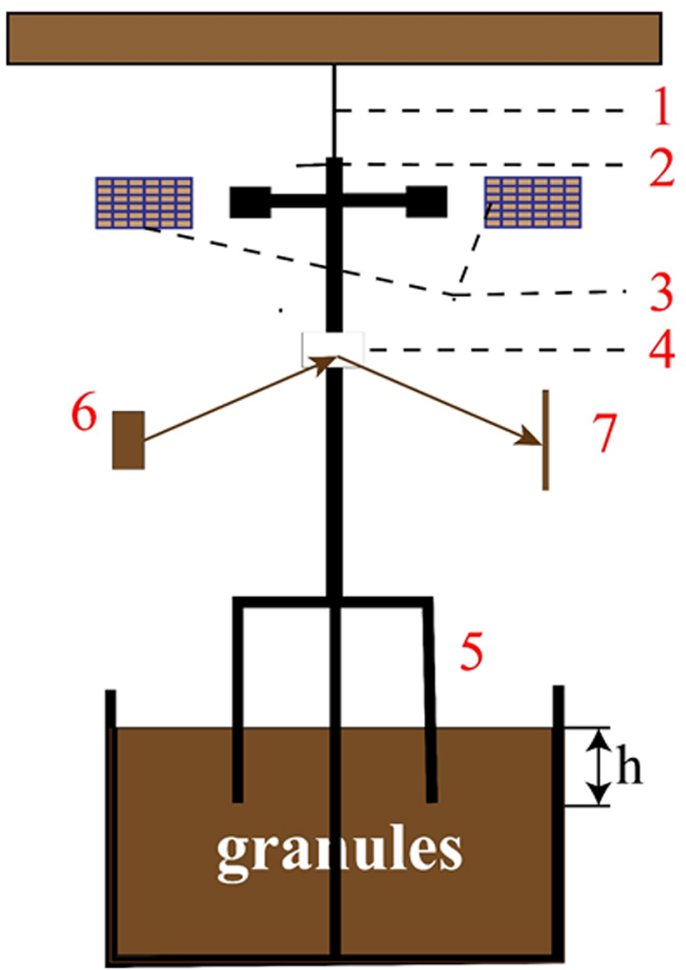

Fig. 1. Sketch of the forced torsion pendulum immersed into a granular material.1, suspension wires; 2, permanent magnet; 3 , external coils; 4 , mirror; 5 , cylinder (made of aluminum alloy with inner radius R) covered or not by a fixed layer of granules; 6 , optic source; 7 , optic detector
$A(t)=A \sin (\omega t-\phi)$, where $\phi$ is the phase difference between $F(t)$ and $A(t)$. A detailed description of the apparatus can be found elsewhere ${ }^{21}$. According to this measurement technique, the relative energy dissipation $\tan \phi$ is recorded by calculating the loss angle between the applied stress and strain. Meanwhile, the shear modulus $G$ is calculated from the ratio between the stress and strain.

In our measurements, we used three different millimeter size glass grains with the diameters (D) of $0.1 \pm 0.05$, $0.5 \pm 0.05$ and $1.9 \pm 0.05 \mathrm{~mm}$. The glass beads are spherical with smooth surfaces. Furthermore, the cylinder (with inner diameter of $24.06 \mathrm{~mm}, 1.11 \mathrm{~mm}$ thickness and $34.92 \mathrm{~mm}$ height), covered by a layer of glued grains with the same size of the investigated granular system, immersed into a large assembly of granules held in a large enough container (with inner diameter of $70.60 \mathrm{~mm}, 8.34 \mathrm{~mm}$ thickness and $39.46 \mathrm{~mm}$ height). Our experiments were conducted on an antivibration table in the natural environment, so that we can ignore vibration-induced effects. To eliminate the effects of the construction history, the cylinder is pushed to a certain depth in the granular assembly. Before each experiment, the granular system is flattened and vibrated by external vibrations to ensure the accuracy of the measurement. Then, all measurements performed at $4.0 \mathrm{~Hz}$, which is below the inherent frequency of the pendulum (about $36.0 \mathrm{~Hz}$ ). During the experiment, an external pressure is increasingly exerted on the medium from the top surface. The arc length amplitude (angular displacement amplitude times radius of the probe) is below $8.0 \times 10^{-5} \mathrm{~m}$ and less than the particle diameter. So the system can be considered as quasistatic.

\section{Results and Discussion}

In the present experiments, two parameters are changed: the immersed depth $h$ and the shear strain amplitude $A$. The energy dissipation $\tan \phi$ and the apparent shear modulus $G$ are analyzed as a function of these two parameters, for granular systems with different grain sizes under pressures. Fig. $2 \mathrm{a}$ and $2 \mathrm{~b}$ show a typical mechanical spectrum $(\tan \phi$ and $G$ ) of the glass beads system with a diameter of $0.1 \pm 0.05$ $\mathrm{mm}$ at different immersed depths. It can be observed that at a small $h$ below $6.94 \mathrm{~mm}$ (about 70 times the diameter of particle), both the $G$ and $\tan \phi$ show small values and decreases with increasing the shear amplitude. The system shows a shear thinning behavior and this indicates the system is in a liquid-like state. When $h$ increases up to $6.94 \mathrm{~mm}$, a strain-dependent loss tangent peak is observed, and at the same time there is a steep decrease in the $G$. This behavior is similar to that observed by Sellerio et al. ${ }^{16}$ and could be ascribed to the jamming transition. As the strain increases, the immersed probe is blocked in the granular medium at first. So the $G$, which is in fact an indicator of the stiffness, is very high. On the contrary, the dissipation is low because the incoming energy tends to be stored in elastic deformation of 


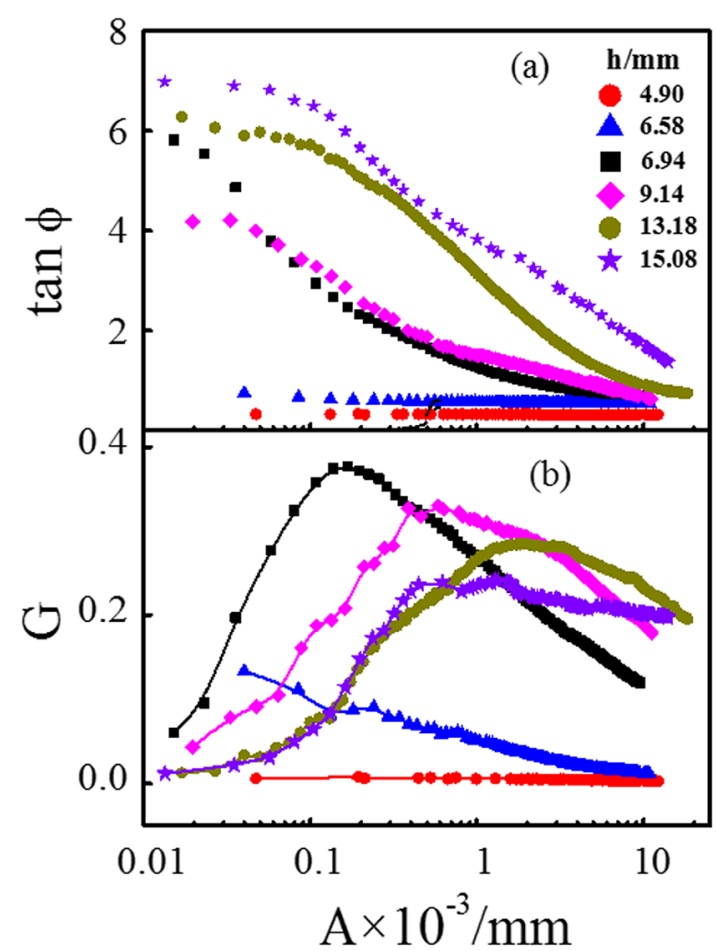

Fig. 2. Typical mechanical spectrum (a) $G$ and (b) $\tan \phi$ of the glass beads with a diameter of $0.1 \pm 0.05 \mathrm{~mm}$ at different immersed depths

the granular network. As the strain is increased, the stiffness has a strong decrease, and the loss tangent shows a peak. With a higher $h$, the dissipation peak shifts to the higher strain, this is because much higher strain/stress is needed to cause the rearrangement of the granular force chain network. As the probe breaks through these force chains, the stiffness decreases, and the dissipation of the energy due to friction between the grains (and between the grains and the surface of the probe) is responsible for the appearance of the peak in the loss tangent.

To get a deeper insight into the dynamics of granular systems, we investigated the dependence of the immersed depth on the energy dissipation at different shear strain amplitude A, as shown in Fig. 3. At first the energy dissipation $\tan \phi$ is low and increases smoothly with the increasing depth $h$ at $h<6.4 \mathrm{~mm}$. In this situation, the probe is free to rotate and the energy dissipation is low. This indicates that the system is in a stable fluidization state. As $h$ further increases, the $\tan \phi$ increases sharply and a dissipation peak appears, which indicates the glass transition arising from jamming. This is understandable because more energy is dissipated due to the friction between the grains when the rotating probe destroys the force chains formed under gravity. The dissipation peak seems to shift to higher $h$ and become lower at a higher $A$. When $h$ increases above $15.0 \mathrm{~mm}$, the $\tan \phi$ shows insensitive to $h$. At this time, the probe is highly blocked and the rearrangement still occurs but slows down, and the system behaves more and more like an amorphous

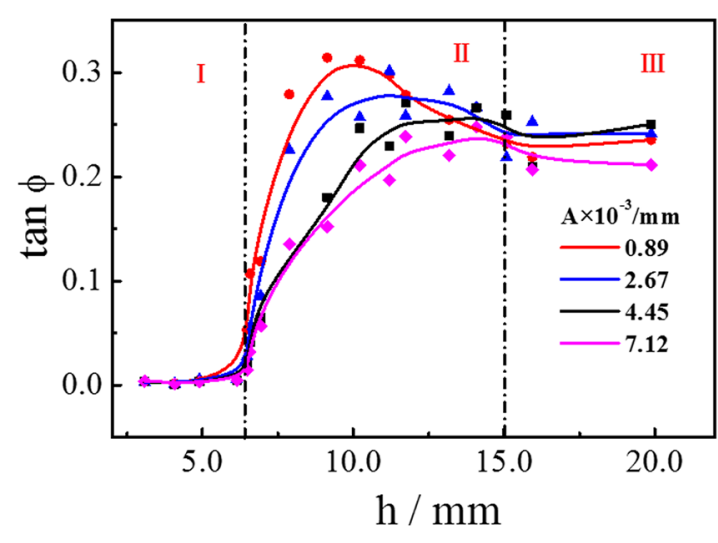

Fig. 3. Dependence of the immersed depth $h$ on the $\tan \phi$ for glass beads with a diameter of $0.1 \pm 0.05 \mathrm{~mm}$ at different shear strain amplitude $A$

solid. Therefore, with increasing $h$, the granular system undergoes a fluid-jamming-frozen transition.

The mechanical responses of granular systems with different particle diameters of $0.5 \pm 0.05$ and $1.9 \pm 0.05 \mathrm{~mm}$ are also examined. The systems show a similar phenomenon as described in Fig. 2 with increasing the shear strain amplitude. Fig. 4 shows the mechanical spectra $(G$ and $\tan \phi)$ of the glass beads systems with different particle diameters as a function of the immersed depth $h$, where the shear amplitude $A=4.45 \times 10^{-3} \mathrm{~mm}$. It can be observed that the value of $G$ increases slowly at first and then strongly with the increasing

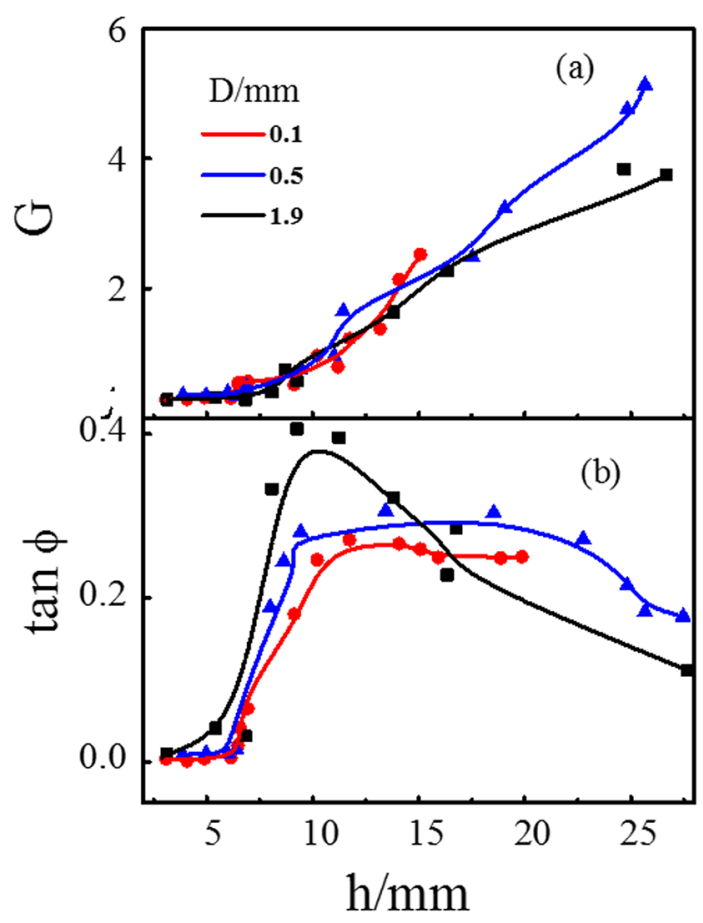

Fig. 4. (a) $G$ and (b) $\tan \phi$ of the glass beads systems with different particle diameters as a function of the immersed depth $h$, where the shear amplitude $A=4.45 \times 10^{-3} \mathrm{~mm}$ 
$h$. At the same time, the $\tan \phi$ shows a peak for all the systems. Note that both the $\tan \phi$ peak and $G$ curve shifts to higher $h$ when the particle diameter is decreased. This effect could be interpreted in this way. As we know, the total contact surface between the probe and the granular medium increases when the particle diameter decreases at the same immersed depth. Therefore, much more force chains formed per unit volume under gravity for smaller particle packings. And a larger stress/strain is needed to cause the arrangement of the force chains and more energy is dissipated during the breakup of the chains when the probe shears back and forth. In other words, when the shear amplitude is the same, a system with bigger particles would exhibit the same $G$ and $\tan \phi$ as a system with smaller particles at lower immersed depth $h$.

Besides the effect of the particle diameter, an important point concerns the structure change of a system with the external pressure. Fig. 5 shows the effect of external pressure on the $G$ and $\tan \phi$ curves of glass beads as a function of $h$. As we change the pressure from 0 to $630 \mathrm{~Pa}$, the curve of shear modulus $G$ shifts slightly to a low immersed depth $h$. At the same time, the $\tan \phi$ peak also moves to a low $h$ a little. This is understandable because the packing fraction of the grain chains increases and the stiffness of the grain chain increases when the chains are in close contact under a high pressure. The effect of pressure is similar to that with increasing the particle diameter.

For further understanding the phenomena observed above, a qualitative explanation is tentatively proposed, as shown in Fig. 6. As we know, the glass grains in the container form

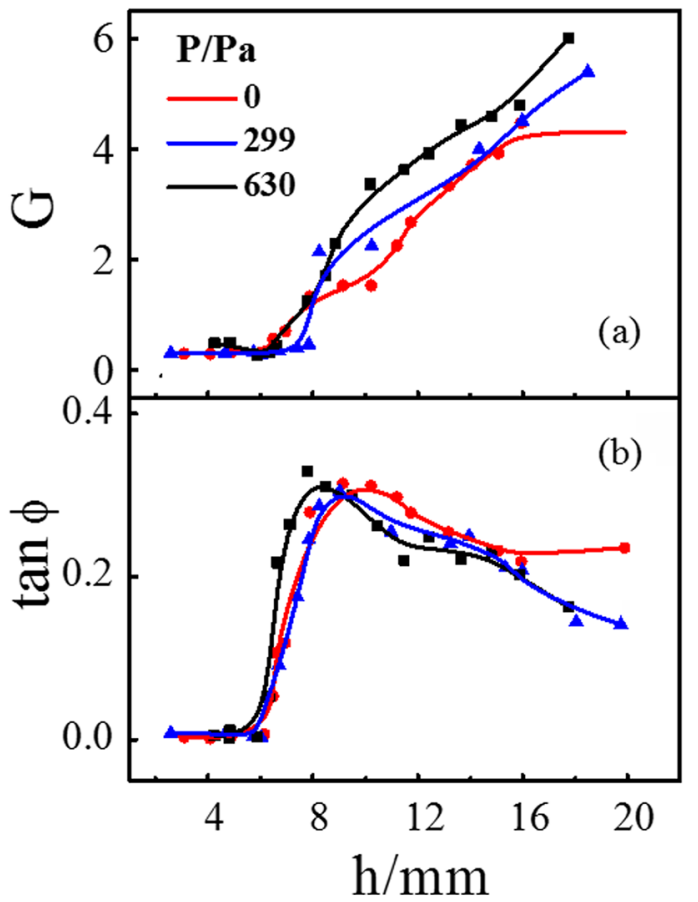

Fig. 5. (a) $\mathrm{G}$ and (b) $\tan \phi$ of glass beads with different external pressures as a function of the immersed depth $h$, where $A=8.90 \times 10^{-3}$ many static inhomogeneous distribution force chains due to the gravity ${ }^{23}$. These chains are terminated on the side surface of rotational cylinder. When cylindrical probe rotates, the grain chains in contact with the cylinder surface received a shear oscillation strain. Here the effect of the increasing amplitude and the decreasing depth on stress chains of the network is considered. While a shear strain is applied to the grain chains, the glass beads that are in contact with each other undergo contact occur inelastic deformation, which has been demonstrated to follow the nonlinear Hertz's law ${ }^{22}$. The glass beads adjust their relative position and take some transversal motion to maintain mechanical balance ${ }^{20}$. If the shear strain is not large enough to collapse the grain chains, the deformation and relative displacement between grains increase with increasing strain. However, the grain chains maintain mechanical balance. So the energy dissipation of relative motion of glass beads is very small and nearly constant compared to that of break-up and rearrangement of grain chains, and the system displays a frozen state.

When the shear strain is increased, the relative transversal displacement of the glass beads becomes larger so that some grain chains will break. These chains vary in lengths and directions. Some grain chains are easy to break; some grain chains are difficult to break. Note that the grain chains vary in lengths and directions, and each chain has its own critical shear strain above which it will collapse. So the critical shear strain also has a distribution and there is a range of shear amplitude between which the chains break. For simplicity, we define the threshold strain corresponding to the beginning of break-up of the grain chains as $\varepsilon_{\text {min }}$, and that associated to the last broken chains as $\varepsilon_{\text {max }}$. When the shear strain is located at $\left[\varepsilon_{\text {min }}, \varepsilon_{\text {max }}\right]$, the strain is enough to cause the rearrangement and reconfiguration in the grain chain network, which leads to the increase of energy dissipation. So the observed $\tan \phi$ peak together with a sharp decrease in the $G$ indicates the onset of jamming. As the strain increases up to $\varepsilon_{\max }$, almost all the grain chains could not stand the shear strain. The grains undergo relatively large slippage with each other and the chains collapse. Then the system behaves like a fluid.

In addition, the effect of increasing the immersed depth on the transitions is just to the opposite of the effect of decreasing

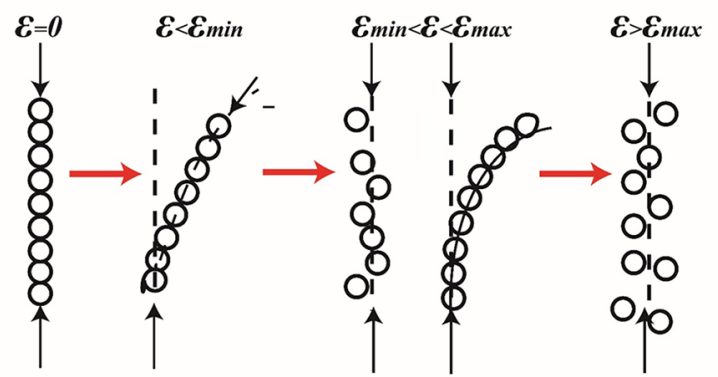

Fig. 6. Schematic of the evolution of strain chain of glass beads with increasing shear amplitude or immersed depth of the probe ( $\varepsilon_{\min }$ and $\varepsilon_{\max }$ is the critical shear amplitude or immersed depth) 
the strain amplitude. Here the maximum immersed depth is about $25 \mathrm{~mm}$, which is less than the characteristic pressure saturation length $\lambda \sim 2 d \sim 69 \mathrm{~mm}$ ( $d$ is the inner diameter of the container) determined by the Janssen model ${ }^{6,22}$. So the granular pressure can be treated as hydrostatic ${ }^{22}$ and it increases with increasing $h$. Therefore, at the same shear stress/strain, the vibration of the probe with a higher $h$ is blocked and the force chains are difficult to rearrange while they are easy with a lower $h$. So with increasing $h$, the system undergoes a fluid-jamming-frozen transition, which is just the opposite to the frozen-jamming-fluid transition with increasing strain amplitude. This is why the effect of increasing the depth on the transitions is equivalent to reducing the shear strain. Therefore, with increasing the shear strain amplitude or decreasing the immersed depth of the probe, the granular system displays three different states: frozen, jamming and fluidized.

\section{Conclusions}

In conclusion, the dynamics of granular systems with different particle diameters has been investigated by lowfrequency mechanical spectroscopy under shear strain and external pressures. With increasing the immersed depth of the oscillating probe, all the systems show the frozen, jamming and fluidized behaviors. The dissipation peak associated with the jamming transition is observed, which shifts to a higher immersed depth when the particle diameter is decreased but moves to lower depth as pressure is increased. A qualitative explanation is proposed to explain the dynamic behaviors of the granular systems based on the change of force chains under shear strain, which determines the state under shear strain or immersed depth.

\section{Acknowledgements}

This work was financially supported by National Natural Science Foundation of China (Nos. 11374298 and 11174283) and Youth Innovation Promotion Association of CAS (2015384).

\section{References}

1. Aranson IS, Tsimring LS. Patterns and collective behavior in granular media: Theoretical concepts. Reviews of Modern Physics. 2006;78(2):641-692.

2. Bi D, Zhang J, Chakraborty B, Behringer RP. Jamming by shear. Nature. 2011;480:355-358.

3. Umbanhowar P, van Hecke M. Force dynamics in weakly vibrated granular packings. Physical Review E. 2005;72(3):030301.

4. Trujillo L, Torres V, Peniche F, Sigalotti LG. Towards a Mathematical Model for Elastic Wave Propagation in Granular Materials. Engineering. 2012;4(12A):972-979.
5. Trappe V, Prasad V, Cipelletti L, Segre PN, Weltz DA. Jamming phase diagram for attractive particles. Nature. 2001;411:772-775.

6. Boutreux T, Raphaël E, de Gennes PG. Propagation of a pressure step in a granular material: The role of wall friction. Physical Review E. 1997;55(5):5759.

7. Pastore R, Ciamarra MP, Coniglio A. 'Flow and jam' of frictional athermal systems under shear stress. Philosophical Magazine. 2011;91(13-15):2006-2013.

8. Ciamarra MP, Pastore R, Nicodemi M, Coniglio A. Jamming phase diagram for frictional particles. Physical Review E. 2011;84(4):041308.

9. Pastore R, Ciamarra MP, Coniglio A. Absence of 'fragility'and mechanical response of jammed granular materials. Granular Matter. 2012;14(2):253-258.

10. D'Anna G, Gremaud G. Activated-like hopping transition in weakly vibrated granular media. EPL. 2001;54(5):599.

11. D'Anna G, Gremaud G. Vibration-induced jamming transition in granular media. Physical Review E. 2001;64(1 Pt 1):011306.

12. D’Anna G, Gremaud G. Vogel-Fulcher-Tammann-type diffusive slowdown in weakly perturbed granular media. Physical Review Letters. 2001;87(25):254302.

13. D'Anna G, Gremaud G. The jamming route to the glass state in weakly perturbed granular media. Nature. 2001;413(6854):407409 .

14. Sellerio AL, Mari D, Gremaud G. Fluidized States of Vibrated Granular Media Studied by Mechanical Spectroscopy. Solid State Phenomena. 2012;184:422-427.

15. Sellerio AL. Towards the Glass Transition in Vibrated Granular Matter. Thesis. Lausanne: École Polytechnique Fédérale de Lausanne; 2012.

16. Sellerio AL, Mari D, Gremaud G, D'Anna G. Glass transition associated with the jamming of vibrated granular matter. Physical Review E. 2011;83(2):021301.

17. Wu X, Zhu Z. Dynamic Crossover of $\alpha$ ' Relaxation in Poly (vinyl acetate) above Glass Transition via Mechanical Spectroscopy. The Journal of Physical Chemistry B. 2009;113(32):11147-11152.

18. Zhou X, Wu X, Wang H, Liu C, Zhu Z. Phase diagram of the Pluronic L64-H2O micellar system from mechanical spectroscopy. Physical Review E. 2011;83(4):041801.

19. Wang H, Wu X, Zhu Z, Liu CS, Zhang Z. Revisit to phase diagram of poly( $\mathrm{N}$-isopropylacrylamide) microgel suspensions by mechanical spectroscopy. The Journal of Chemical Physics. 2014;140(2):024908.

20. Xiong XM, Zhang JX. Amplitude dependence of elasticity for the assembly of $\mathrm{SiO} 2$ powders under shear oscillation strain. Physical Review E. 2010;81(4):042301.

21. Wang WJ, Kong XZ, Zhu ZG. Friction and relative energy dissipation in sheared granular materials. Physical Review E. 2007;75(4 Pt 1):041302.

22. Chai L, Wu X, Liu C. A universal scaling law of grain chain elasticity under pressure revealed by a simple force vibration method. Soft Matter. 2014;10(35):6614-6618.

23. Peters JF, Muthuswamy M, Wibowo J, Tordesillas A. Characterization of force chains in granular material. Physical Review E. 2005;72(4):041307. 